\title{
Extended Modelica Model for Heat Transfer of Two-Phase Flows in Pipes Considering Various Flow Patterns
}

\author{
Timm Hoppe $^{1}$ Friedrich Gottelt $^{1} \quad$ Stefan Wischhusen $^{1}$ \\ ${ }^{1}$ XRG Simulation GmbH, Harburger Schlossstr. 6-12, 21079 Hamburg Germany, \\ \{hoppe, gottelt, wischhusen\}@xrg-simulation.de
}

\begin{abstract}
Boiling in vertical and horizontal pipes is a complex process defining transient and static performance of various technical applications. This work presents an extended heat transfer model which takes the complete boiling process into account. Models from the literature for the different boiling regimes are evaluated with respect to accuracy and suitability for system simulation application. A set of sub-models for each of the existing boiling phenomena is implemented and applied to the global boiling model. Special attention is paid to smooth transition between the sub-models and to numerical efficient solutions with respect to the consideration of the boiling crisis. The simulation results show good accordance with literature data.

Keywords: boiling model, heat transfer, two phase flow, pipe flow, evaporation, critical heat flux, boiling crisis, subcooled boiling, saturated boiling, flow pattern, FluidDissipation, ClaRa
\end{abstract}

\section{Introduction}

The heat transfer from an evaporator wall to a flowing two-phase fluid is called flow boiling. During flow boiling three different regimes can be identified. The first one is the subcooled boiling regime. The bulk of the fluid is still subcooled but bubbles can already form in the wall layer, are cooled by the surrounding liquid and thus enhance the heat transfer. It is followed by the saturated boiling regime in which the wall is predominantly covered by the liquid phase. Bubbles are formed there, leave the wall and exchange heat and mass with the surrounding liquid at saturation temperature. The critical heat flux marks the beginning of the post critical heat flux regime, where the gas phase becomes the dominating phase in the wall layer. In all of these regimes the slope of the pipe plays an important role. For example, a vertical pipe has a distinct point at which the regime changes from saturated boiling to the post critical heat flux regime. In a horizontal pipe there is a gradually transition, as the top of the pipe may be already covered by steam, while the bottom of the pipe is still cooled by liquid.

During flow boiling of fluid flows high heat fluxes can be transferred at low temperature differences. Flow boiling occurs in many industrial applications, such as thermal power plants, air conditioning or heat exchangers in the process industry. Exact knowledge about the two-phase heat transfer and pressure loss is important to determine behaviour of these applications. However, it is quite common to reduce the boiling process to the saturated boiling regime in system simulation, although several boiling regimes can be identified which require specific models. This assumption neglects important effects like the critical heat flux at which the heat transfer coefficient drops by magnitudes.

An industrial example where it is important to include this effect are different kinds of thermal power plants like conventional coal fired steam generators, solar steam generators, natural circulation heat recovery steam generators or nuclear steam generators.

In coal fired once-through steam generators the critical boiling state occurs during normal operation at the end of the evaporator. In normal operation the mass flow rate is high enough to sustain a sufficient cooling of the pipes, see (Brinkmeier, 2015). However, in abnormal working conditions, e.g. maldistribution of mass flow between parallel pipes or failure of feed water supply systems the mass flow rate may be significantly lower than during nominal operation. A detailed calculation of the heat transfer is needed to determine the wall temperatures in this abnormal situations.

\section{State of the Art of Two-phase Heat Transfer Modelling}

\subsection{Literature Review}

The actual steam quality of the flow is described by the local vapour mass fraction defined by the ratio of vapour mass flow $\dot{m}_{\text {vap }}$ to total mass flow $\dot{m}$ :

$$
x_{a c t}=\frac{\dot{m}_{\text {vap }}}{\dot{m}}
$$

If thermodynamic equilibrium is assumed the steam quality can as well be defined by the local specific enthalpy $h$, the specific enthalpy at bubble point $h^{\prime}$ and the specific evaporation enthalpy $\Delta h_{V}$ :

$$
x_{e q} \equiv x=\frac{h-h^{\prime}}{\Delta h_{V}}
$$

The thermodynamic equilibrium steam quality is used in the further course of the paper and will be referred to as 
$x$. In the subcooled area it can also be negative. The overall boiling process with the different boiling regimes is displayed schematically for a horizontal pipe in Figure 1. Two different heating situations are shown, one with a high heat flux resulting in a nucleate boiling dominated flow and one with a low heat flux resulting in a convective boiling dominated flow. The circumferential averaged heat transfer coefficient is plotted against the actual steam quality and the steam quality assuming thermodynamic equilibrium.

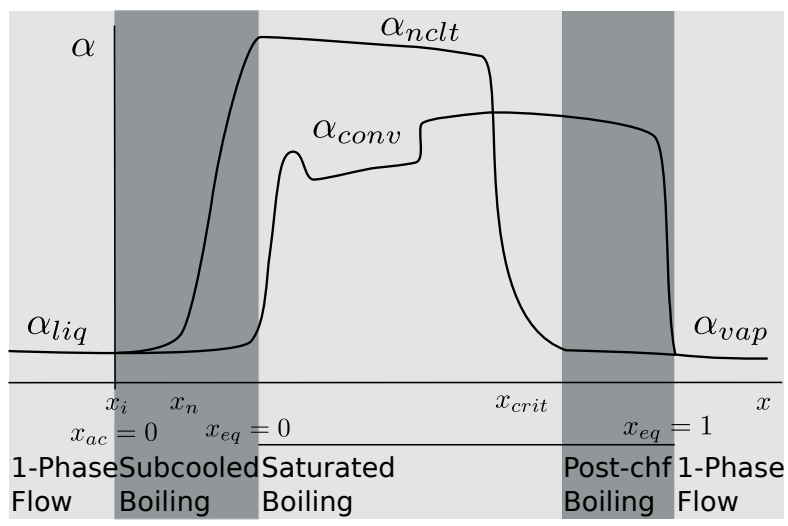

Figure 1. Schematic circumferential averaged heat transfer coefficient for an evaporating flow in a horizontal pipe with a high heat flux (nucleate boiling dominated) and a low heat flux (convective boiling dominated), according to (Steiner, 2002), flow regime descriptions refer to nucleate boiling dominated flow

\section{Subcooled Boiling}

Bubble formation starts in the wall layer of the current already at bulk enthalpies below the bubble enthalpy, i.e. $x<0$. These bubbles deteriorate as they move to the subcooled core of the flow, thus enhancing the heat transfer coefficient. This is known as subcooled boiling, see Figure 1 for a schematic behaviour of the heat transfer coefficient in the subcooled boiling regime of a nucleate boiling dominated flow.

In general the literature on subcooled boiling is sparse compared to other boiling regimes. A comparison of several subcooled boiling models was done by Spindler (K. Spindler, 1990), the model proposed in the VDI heat atlas (Schröder, 2002) shows the least error in prediction of the measurements. At the end of the subcooled boiling regime at a steam quality $x=0$ the VDI model is by construction equivalent to the saturated boiling heat transfer model for a vertical pipe from the VDI heat atlas. Therefore, a smooth transition to the following boiling regime is ensured.

\section{Saturated Boiling}

In the saturated boiling regime two different boiling modes can be observed. This is convective boiling on the one hand and nucleate boiling on the other hand. Convective boiling describes the convective process between the wall and the liquid phase, whereas nucleate boiling describes the heat transfer induced by formation, growth and departure of the bubbles. In horizontal pipes a stratification of the fluid can occur, depending on the present flow pattern. As the upper pipe wall is partly dry, i.e. only covered by the gaseous phase, the circumferential heat transfer coefficient is lower compared to flow patterns which cover the wall completely with the liquid phase.

All relevant saturated boiling models are a function of the convective boiling heat transfer coefficient, basically calculated with convective one phase heat transfer correlations and the nucleate boiling heat transfer coefficient, basically calculated with pool boiling heat transfer correlations. A simple addition of the coefficients is done by the Chen model (Chen, 1966). He introduced a boiling surpression factor and a two phase multiplier to fit pool boiling heat transfer correlation and the one phase convective heat transfer correlation to his flow boiling data. Shah (Shah, 1982) proposed a model which is not superimposing the convective and the nucleate heat transfer coefficients but chooses the larger of the two. Gunger and Winterton (Winterton, 1986) developed a new form of the Chen model, basing on a larger database. They later also proposed a simpler version of the model (Gungor and Winterton, 1987). The VDI heat atlas (Steiner, 2002) proposes an asymptotic model which incorporates natural limitations of the flow boiling coefficients, instead of surpression and two phase factors as used by the other models. It is also refered to as the Steiner-Taborek model as it bases on a publication of the two authors (Steiner and Taborek, 1992). The model is recommended by ASHRAE (Owen, 2005) and Thome (Thome, 2006a).

\section{Critical Heat Flux}

In the further course of the evaporation process the critical boiling state $x_{\text {crit }}$, also known as critical heat flux, is reached and the gas phase becomes the dominating phase at the wall. In a vertical pipe it is a distinct point at which the heat transfer coefficient drops suddenly by magnitudes. In a horizontal pipe the critical boiling state at the top of the pipe can already be reached while the bottom of the pipe is still covered with liquid. The circumferential averaged heat transfer coefficient in such a situation can be seen in Figure 1.

Several approaches for prediction of the critical heat flux can be found in literature. This are on the one hand the local hypothesis correlations which take the local conditions at the point of boiling crisis into account. Then there are the global hypothesis correlations which consider the conditions at the pipe inlet. A third method are the look-up tables.

The VDI heat atlas (Auracher et al., 2002) gives the Groeneveld tables (Groeneveld, 2007) as reference for look-up tables and the Katto-Ohno model (Katto Y., 1984) as reference for the global hypothesis correlations. The Katto-Ohno correlation is also proposed by Thome (Thome, 2006a). For medium to high pressures and mass flow rates the VDI heat atlas proposes the local hypothesis correlations of Doroshchuk and Kon'kov. They are con- 
sidered as superior to the other two methods. Furthermore, they differentiate between dry out and departure from nucleate boiling.

\section{Post Critical Heat Flux Boiling}

At least two boiling regimes can be recognized after the critical boiling state. On the one hand there is the dry out of the surface. The wall is not necessarily dry, as the remaining liquid is entrained as droplets in the vapour flow, also referred to as mist flow. Droplets may then hit the wall and wet it temporarily. The second regime is called film boiling or departure from nucleate boiling. It is characterized by the formation of a gas film, the corresponding flow pattern is called inverted annular flow. The heat transfer coefficient is significantly lower than in mist flow. For each regime different heat transfer models are required. If the wall temperature is very high compared to the gas temperature radiation will also play an important role in the heat transfer.

For dry out there are two different kinds of correlations. The simpler correlations assume thermodynamic equilibrium between gas and liquid phase. The model of Groeneveld (Groeneveld, 1973) is proposed by the VDI heat atlas (Katsaounis, 2002) and by Thome (Thome, 2006a).

The more complicated models account for thermodynamic non-equilibrium effects by an apparent superheated gas temperature. The Köhler model (Köhler, 1983) proposed by the VDI heat atlas is to mention here. A model which also includes radiation was published by Ganic and Rohsenow (Ganic and Rohsenow, 1977).

\section{Summary}

The VDI heat atlas proposes correlations for all different boiling mechanisms, thus the complete boiling process is covered by the VDI proposal. In some extent the models refer to each other. For example one model merges by construction into the model for the following boiling regime. This smooth transition between the different models is important in system simulation. It should be continuous, continuous differentiable, with low gradients and without hysteresis. The proposed models are also considered among the most reliable ones by other authors. Therefore, the models of the VDI heat atlas are implemented. Where it is possible the references between the models are used to smooth or simplify the transitions.

\subsection{Library Review}

It is not known to the authors that an existing Modelica library incorporates a heat transfer model for the complete boiling process. The AirConditioning library and the TIL library, which are commercially used for simulation of automotive refrigeration cycles, cover only the saturated boiling regime. The AirConditioning includes implementations of the simple Gungor-Winterton and the Chen correlation. The TIL library includes the Chen correlation and the convective boiling correlation of the Steiner-Taborek model. In the freely available ThermoCycle library the Shah and the simple Gungor-Winterton correlation are im- plemented.

In power plant modelling the critial heat flux plays an important role. In the freely available ClaRa library only the saturated boiling regime is covered. Also other libraries, the freely available ThermoSysPro, the freely available ThermoPower and the commercial ThermalPower, provide no correlations for determination of the boiling crisis.

\section{Implementation}

The different boiling regime correlations are implemented in the FluidDissipation which is an open source library and freely available, see (XRG Simulation). For implementation the functional approach described in (Vahlenkamp and Wischhusen, 2009) is used. The main aspects of the approach are:

\section{- Independence of thermo-hydraulic framework}

- Use of function calls

- Inputs are delivered by records

In contrast to the models already implemented in the FluidDissipation each boiling regime is implemented in a separate model. Thus, the overall heat transfer models are built outside of the FluidDissipation. The advantage of this procedure is that models can be developed which are tailored to the corresponding problem. In the following the implementation of the separate models is shortly described focussing on main declarative equations and differences of the implementation to the VDI heat atlas models. For detailed description of the models see the FluidDissipation documentation.

\subsection{Subcooled Boiling Heat Transfer}

From the VDI heat atlas (Schröder, 2002) the models for determination of the position, in terms of a steam quality, of initial bubble formation $x_{i}$ and of net vapour forming $x_{n}$ are implemented. Both values are by definition of the subcooled boiling regime always negative. For $x_{i}<x<x_{n}$ the proposed model for calculation of the heat transfer coefficient from VDI heat atlas is used. For $x_{n}<x<0$ the VDI heat atlas proposes a superposition of the nucleate boiling heat transfer coefficient and the subcooled convective heat transfer coefficient. However, this model incorporates the wall temperature in the heat transfer coefficient calculation, which would introduce additional iterations for the solver. Furthermore, the transition to the saturated boiling heat transfer coefficient is only smooth if the influence of the flow pattern on the saturated boiling heat transfer coefficient is neglected. To simplify this model, thus making it numerical more stable, a simple smoothing from the subcooled convective heat transfer coefficient $\alpha_{s c}\left(x=x_{n}\right)$ to the saturated heat transfer coefficient $\alpha_{\text {sat }}(x=0)$ is done. The Stepsmoother from the FluidDissipation is used which makes use of the smooth transition 
of the following equation

$$
y_{\text {reg }}=(1+\tanh (\tan (x))) / 2
$$

The output $y_{\text {reg }}$ is between 0 and 1 for $\mathrm{x}=[-\pi / 2, \pi / 2]$.

\subsection{Saturated Boiling Heat Transfer}

The Steiner-Taborek model from the VDI heat atlas (Steiner, 2002) is implemented. The following equation results in an asymptotic combination of the convective boiling $\alpha_{\text {conv }}$ and the nucleate boiling heat transfer coefficient $\alpha_{\text {nclt }}$

$$
\alpha_{\text {sat }}=\sqrt[3]{\alpha_{c o n v}^{3}+\alpha_{n c l t}^{3}}
$$

\section{Convective Boiling}

The VDI heat atlas distinguishes between horizontal and vertical pipes, in consequence two different correlations are provided. Both depend on the one phase heat transfer coefficients for liquid and steam $\alpha_{l i q}$ and $\alpha_{v a p}$, the density ratio $\rho^{\prime} / \rho^{\prime \prime}$ and the steam quality $x$. For horizontal pipes the VDI correlation includes a correction for stratified flow patterns. The information of the present flow pattern and the angle of the unwetted circumference of the pipe is needed. The correction weights the one phase liquid and steam heat transfer coefficients according to the unwetted angle. In the implementation of these functions a smoothing with the stepsmoother from the FluidDissipation is applied for the transition between different flow patterns.

\section{Nucleate Boiling}

The used correlation from the VDI heat atlas is a function of heat flux $\dot{q}$, pressure $p$, pipe diameter $d$ and surface roughness $W$. For horizontal pipes the correlation shows an additional dependency on the mass flow rate $\dot{m}$ and on the steam quality $x$. Furthermore, a correction includes the dependency of the present flow pattern. The function expects the present flow pattern of the flow as an input and aligns a correction factor to each flow pattern. These correction factors depend on the thickness and conductivity of the wall. Also in this implementation the transition between flow patterns is smoothed.

\subsection{Boiling Crisis}

The position of the critical boiling state in terms of a critical steam quality $x_{c r i t}$ is determined by the correlations which are explained in this subchapter. The critical steam quality marks the end of the saturated boiling regime and the begin of the post critical heat flux regime and therefore determines in an overall boiling model at which point the heat transfer coefficient calculation has to change from the saturated boiling correlation to the post critical heat flux correlation.

The local thesis correlations of the VDI heat atlas (Auracher et al., 2002) of Konkov and Doroshchuk for water are implemented. They depend on the local mass flow rate, the local pressure, diameter of the pipe and the local heat flow rate.

The Konkov correlation predicts the critical steam quality for dry out, the Doroshchuk correlation the critical steam quality for departure from nucleate boiling. A logic chooses the smaller of the two. The used correlation determines whether dry out or departure from nucleate boiling is present. Thus, it can be decided which post critical heat flux correlation has to be used.

For horizontal pipes gravitational effects have to be considered. With the modified Froude number $\mathrm{Fr}$ these effects can be described

$$
F r=\frac{x_{\text {crit }} \dot{m}}{\sqrt{\rho^{\prime \prime}} \sqrt{9.81 d\left(\rho^{\prime}-\rho^{\prime \prime}\right) \cos (\theta)}} .
$$

The angle $\theta$ is the inclination of the pipe. For Froude numbers $<10$ the stratification of the flow induces an occurrence of the boiling crisis at the upper side of the pipe $x_{c r i t, u p}$ at much lower steam qualities than at the bottom side of the pipe $x_{\text {crit, low }}$. This difference $\Delta x_{\text {crit }}$ can be determined with the modified Froude number

$$
\Delta x_{\text {crit }}=x_{\text {crit }, \text { low }}-x_{\text {crit }, u p}=\frac{16}{(2+F r)^{2}} .
$$

With the difference $\Delta x_{\text {crit }}$ the transition zone in horizontal pipes from the saturated boiling regime to the post critical heat flux regime can be determined.

\subsection{Post Dry-Out Heat Transfer}

Two different kinds of models exist for the post dry out heat transfer. One type assumes thermodynamic equilibrium between the liquid and the vapour phase, i.e. the temperatures are equal. The other type calculates a superheated gas temperature, which determines the temperature difference for the heat transfer.

\section{Thermodynamic Equilibrium}

The proposed model from the VDI heat atlas (Katsaounis, 2002) is implemented. According to the heat atlas the scope of the model is only for large mass fluxes $\dot{m}>2000 \mathrm{~kg} /\left(\mathrm{m}^{2} s\right)$ and high pressures with $\rho^{\prime} / \rho^{\prime \prime} \leq 6$. However, the model bases on data of a much wider range, according to (Thome, 2006b).

\section{Thermodynamic Non-Equilibrium}

The proposed model for thermodynamic non-equilibrium from the VDI heat atlas (Katsaounis, 2002) is implemented. The assumption of the vapour liquid equilibrium is not valid in this boiling regime. The heat transferred from the wall to the fluid is not used completely for vaporising the liquid but also for superheating the gas phase. Thus, the temperature difference for heat flow rate calculation is calculated with the temperature of the superheated gas and the wall temperature within the scope of the model, i.e.:

$$
\dot{Q}=\alpha_{c h f} A\left(T_{w}-T_{g}\right) .
$$


For calculation of the gas temperature the model provides an empirical correlation. It is no result of energy balancing.

The steam quality $x_{l i m}$ gives the end of validity of the model in terms of steam quality and is an output of the model. For $x>x_{\text {lim }}$ the original source of the model (Köhler, 1983) suggests to keep the wall temperatures constant. As the object-oriented modelling approach does not allow a direct manipulation of the wall temperature calculation, this behaviour is approximated implicitly by keeping the superheated gas temperature constant. This temperature is used for calculation of the heat flux until the equilibrium temperature of the fluid is greater.

\subsection{Flow Pattern Map}

The calculation bases on the flow pattern map model described in the VDI heat atlas (Steiner, 2002). To determine the flow pattern the angle of the unwetted circumference of the pipe $\varphi$ is needed. The VDI heat atlas model uses an iteration process to calculate the unwetted angle. To reduce iterations and make the model suitable for system simulations some modifications have been made. The unwetted angle is calculated directly with the approximation suggested by Biberg (Biberg, 1999).

The output of the function is the unwetted angle $\varphi$ and a real variable flowPattern. To each number a flow pattern is aligned to, see Figure 2. The variable flowPattern and the unwetted angle are used by the saturated boiling heat transfer model, see section 3.2. They identify with the value of the variable the present flow pattern and correct the heat transfer coefficient, accordingly. The transition between two flow patterns is smoothed using the FluidDissipation Stepsmoother see, equation 3.

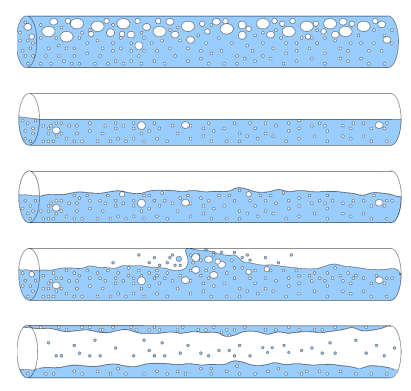

- Bubble flow $\rightarrow 6$

- Stratified flow $\rightarrow 1$

- Wavy flow $\rightarrow 2$

- Slug flow $\rightarrow 3$

- Annular flow $\rightarrow 4$

Figure 2. Flow patterns for two phase flow in horizontal straight pipes.

\section{Application}

In this section an examplary application of the functions is described. The overall boiling process including flow pattern effects is implemented in the model. The combination of the subfunctions is done in a replaceable model applying the FluidDissipation functions. This makes the introduction of additional states possible. These states are necessary due to numerical reasons. The thermo-hydraulic framework of the ClaRa library, (The ClaRa development team) and (Brunnemann et al., 2012), is used. The application is done for the three conservation equation pipe model. The main features of the pipe model are:

- homogeneous single phase, i.e. thermodynamic equilibrium between the phases and same velocities for gas and vapour phase

- one dimensional flow direction

- dynamic energy and mass balances, static momentum balance

- balance equation spatially discretised in flow direction

\subsection{Transition of subfunctions}

Main task of the application model is to ensure a smooth transition between the different heat transfer modes. The smoothing function, defined by equation 3 , is used to ensure a continuous and numerical stable transition. In Figure 3 an example for the transition from saturated boiling to post critical heat flux boiling is given. In the example the transition zone is defined by

$$
t z=\Delta x_{c r i t}
$$

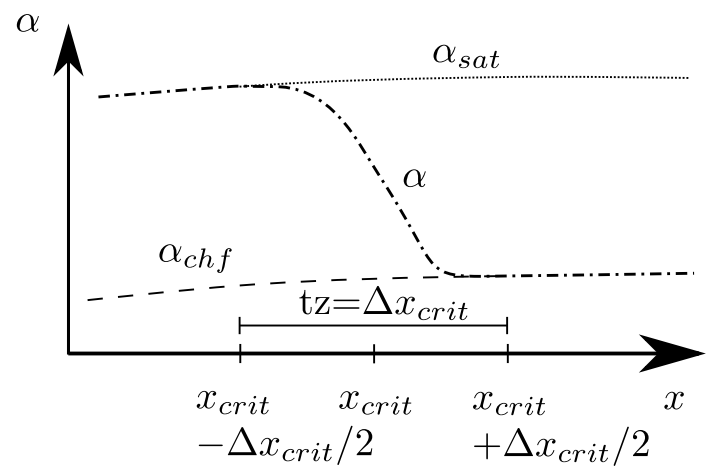

Figure 3. Schematic picure for transition from saturated to post critical heat flux boiling

In the following the used submodels and the switching between the submodels is described in detail. Figure 4 gives an overview of the used models and the selection sequence which is run by the model for determining the heat transfer mode. The corresponding transition zone is displayed in the figure as well. The chosen values of the transition zone are a trade-off between accuracy and numerical performance.

1. Superheated one-phase flow: The one-phase heat transfer model (Dittus-Bölter) from the FluidDissipation is used. The transition zone depends on the choice of the post critical heat flux heat transfer model. For the equilibrium model the transition zone is defined from $x=0.9$ to $x=1$. 


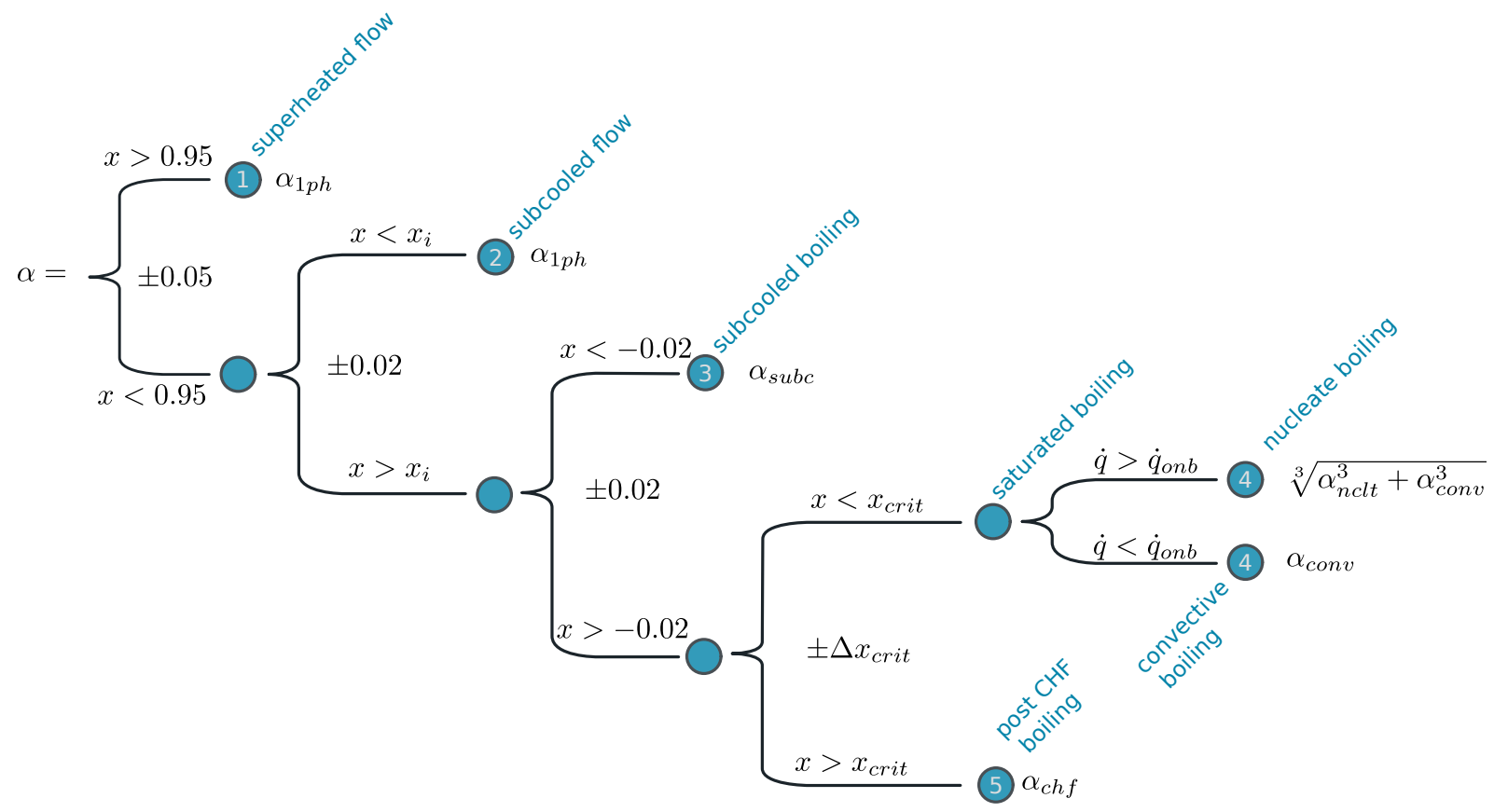

Figure 4. Transition between different Boiling Regimes

2. Subcooled one-phase flow: The one-phase heat transfer model (Dittus-Bölter) from the FluidDissipation is used. The point of initial bubble formation $x_{i}$ is calculated by the subcooled boiling model from section 3.1. The transition zone is defined from $x_{i}-0.02$ to $x_{i}+0.02$.

3. Subcooled boiling: The model for subcooled boiling described in the previous section is used. At $x=0$ the model is merged by construction of the equations into the saturated boiling model described in the previous section.

4. Saturated boiling: The model for saturated boiling described in the previous section is used. If the minimum heat flux for onset of nucleate boiling is exceeded the saturated boiling heat transfer coefficient is calculated according to equation 4 otherwise only convective boiling is present. The critical steam quality $x_{\text {crit }}$ is calculated by the boiling crisis model. The transition area in terms of a steam quality difference is provided for a horizontally oriented tube by the boiling crisis model. For vertically oriented tubes a fixed value of $\Delta x_{\text {crit }}=0.05$ is set. Thus, the transition area is defined by $\mathrm{tz}= \pm \Delta x_{\text {crit }}$.

5. Post-CHF boiling: The models described in the previous section are used. It can be decided whether the thermodynamic equilibrium or the non-equilibrium model is used.

\subsection{Calculation of critical heat flux}

The position of the critical heat flux is calculated using the functions of Konḱov and Doroshchuk (Auracher et al.,
2002) which assume a local hypothesis for the critical heat flux. However, the position is calculated for the total pipe and not for each cell. Thus, situations are avoided in which the model calculates several boiling crises at a time in different cells. Otherwise inconsistent results could be obtained with the previously described selection sequence of the model. As inputs to the critical heat flux model the pressure and mass flow rate of the last cell are used. The heat flow rate is averaged over the total pipe. Thus, a strong numerical coupling between the wall temperatures, heat flows and the heat transfer coefficients of all cells is introduced. To uncouple these variables and help the simulator to break up the resulting non-linear systems of equations a stabilizer state for the heat flow rate $\dot{Q}_{-}$is introduced with the following equation:

$$
\frac{d \dot{Q}_{-}}{d t}=\frac{\dot{Q}-\dot{Q}_{-}}{\tau}
$$

During stationary conditions both heat fluxes are equal, thus no new information is included, the stationary results do not change. During transient conditions the additional state lags behind the real heat flux $\dot{Q}$ with the time constant $\tau=0.1 s$.

In consonance with the calculation of the critical heat flux position also the point of initial bubble formation $x_{i}$ and the point of net vapour generation $x_{n}$ are calculated for the total pipe and not for each cell. The fluid properties of the first cell are handed over to the two models. The average stabilizer state heat flow rate of equation 9 is used as a heat flow rate in the models. 


\section{Verification and Discussion of first Results}

\subsection{Verification of overall heat transfer model}

For verification of the total model which is described in section 4 two simulations of evaporation of water in a horizontal pipe are conducted. A screenshot of the used model built with the ClaRa library is shown in Figure 5. The re-

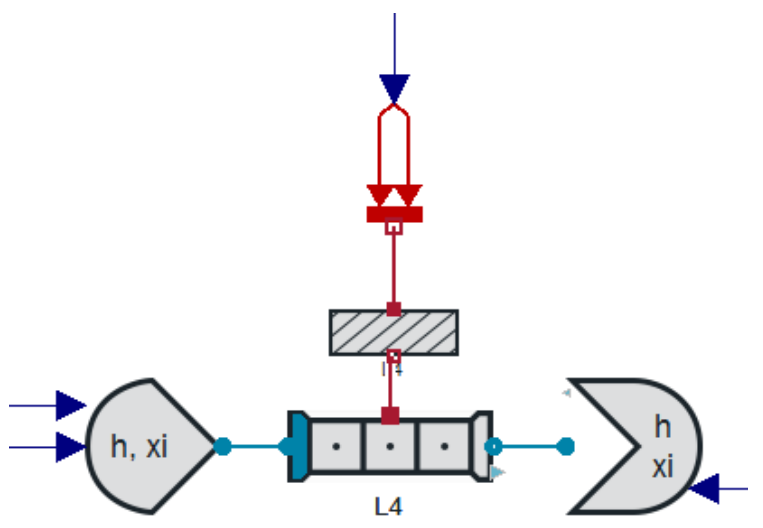

Figure 5. Screenshot of test model

sults should show the principle behaviour of the heat transfer coefficient as can be seen in Figure 1. The boundary conditions and geometric parameters are shown in Table 1, column "Sim. 1". The first values in column one refer to the extreme case of a convective boiling dominated flow, the second values to the extreme case of a nucleate boiling dominated flow. Furthermore, an independency of the flow pattern is assumed, which occurs in pipe walls with a good conductivity (Steiner, 2002). A circumferential uniform heating is assumed. The pipe is discretised with 60 control volumes. The pipe length is chosen such that the fluid is evaporated completely in both cases.

The simulation results are shown in Figure 6. The cir-

Table 1. Boundary conditions and geometric parameters of verification simulation

\begin{tabular}{llll}
\hline Parameter & Sim. 1 & Sim. 2 & Unit \\
\hline Mass flow & $500 / 400$ & 500 & $\mathrm{~kg} /\left(\mathrm{m}^{2} \mathrm{~s}\right)$ \\
Heat flow & $20 / 750$ & 600 & $\mathrm{~kW} /\left(\mathrm{m}^{2}\right)$ \\
Outlet pressure & 50 & 50 & $\mathrm{bar}$ \\
Inlet spec. enthalpy & 550 & 800 & $\mathrm{~kJ} / \mathrm{kg}$ \\
Hyd. diameter & 0.032 & 0.032 & $\mathrm{~m}$ \\
Pipe length & $600 / 15$ & 15 & $\mathrm{~m}$ \\
Wall conductivity & high & low & - \\
\hline
\end{tabular}

cumferential averaged heat transfer coefficient is plotted against the steam quality. The convective boiling dominated case shows a behaviour which is to be expected from Figure 1. The heat transfer coefficient rises with a steam quality of zero, thus no subcooled boiling is present. It rises further with rising steam quality until it peaks at a

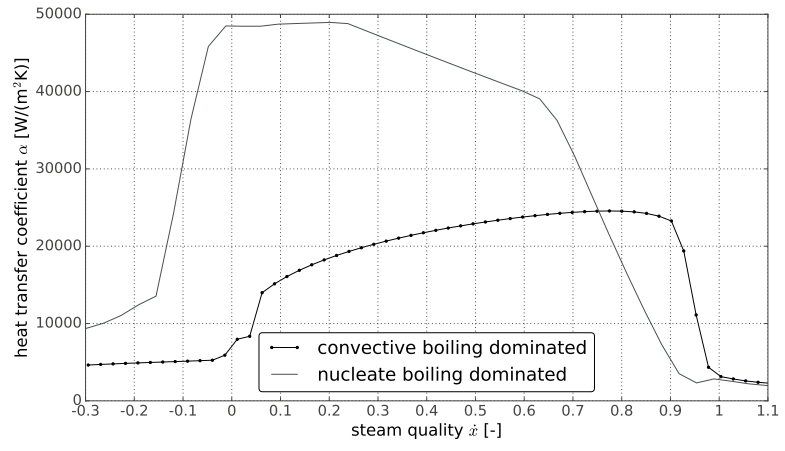

Figure 6. Simulation results of evaporation of water in a horizontal pipe

steam quality of $x=0.8$. From that point on the heat transfer coefficient drops until the one phase heat transfer at a steam quality of 1 is reached, thus no post critical heat flux boiling is present. The nucleate boiling dominated case shows a distinct subcooled boiling regime. A plateau of the heat transfer coefficient follows at which a limit of the effect of mass flow is reached, according to the nucleate boiling model from the VDI (Steiner, 2002). After that plateau the nucleate boiling heat transfer coefficient drops as one could expect from the schematic Figure 1. At a steam quality of $x=0.65$ the critical heat flux at the upper side of the pipe is reached. At $x=0.9$ the critical heat flux is reached also at the bottom side, a post critical heat flux regime follows.

In summary it can be said that the results for both extreme conditions are in very good consonance with the schematic figure. The model predicts the occurrence of the different heat transfer regimes correctly. Also the transition between the different subfunctions is handled in a plausible way.

\subsection{Comparison with a simple saturated boil- ing heat transfer model}

A simulation with the boundary conditions from Table 1, column "Sim. 2", is conducted with the overall heat transfer model described in section 4 and the GungorWinterton 1986 (Winterton, 1986) heat transfer model which is widely used in system simulation. The boundary conditions are chosen such that they lie in between the extreme conditions of a nucleate and a convective dominated flow. Furthermore, a pipe with a low thermal conductivity of the wall is used.

The results are shown in Figure 7. As an interpretation of the results a schematic pipe with the present flow pattern is drawn below the plot. The flow pattern is calculated from the functions described in section 3.5. The overall heat transfer model predicts a distinct subcooled boiling regime. This boiling regime is neclected in the Gungor-Winterton model and the one phase heat transfer coefficient is used instead. Thus, the heat transfer coefficient of the overall model is over a wide range multiple times larger than the one of the simple Gungor-Winterton 


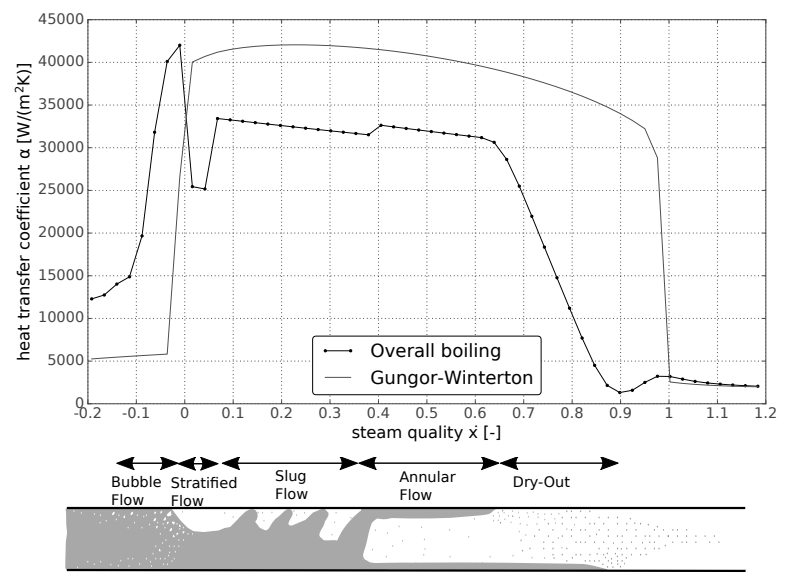

Figure 7. Simulation results of evaporation of water in a horizontal pipe

model.

At steam qualities slightly below zero the heat transfer coefficient of the overall model peaks before it drops again at steam qualities larger than zero. This is due to the fact that the subcooled boiling model does not depend on the flow pattern. Whereas with begin of saturated boiling the heat transfer coefficient shows a high dependency on the flow pattern. At low steam qualities a stratified flow is present. As the upper surface of the pipe is not wetted due to stratification, the circumferential averaged heat transfer coefficient is significantly lower than the GungorWinterton model. It predicts a up to $60 \%$ larger heat transfer coefficient, as it has no correction due to stratification effects of the flow.

In the further course of the evaporation process the overall boiling model predicts two more different flow patterns. Both induce only a partial wetting of the upper pipe wall, thus the heat transfer coefficient is reduced compared to the simple Gunger-Winterton model.

In the overall heat transfer model the saturated boiling regime ends at a steam quality of $x=0.65$. The dry out begins at the upper side of the pipe. At the end of the dry out process at a steam quality of $x=0.9$ the heat transfer coefficient is approximately 10 times smaller than predicted by the simple Gungor-Winterton model.

The results show that the neglection of effects can lead to over- or underestimation of the heat transfer coefficient by magnitudes. For applications, in which crucial variables depend strongly on the heat transfer coefficient, simple models fail to produce reliable results. For example, this may be the case for wall temperatures in the post dry out regime. In that case it is important to include more complex heat transfer models which cover the overall boiling process.

\subsection{Validation with measurements}

As a validation case the Becker experiments (Abel-Larsen et al., 1985) are presented. They are a series of steady state critical heat flux experiments, all of them with a dry-out heat crisis. The test section is a vertical pipe, electrically heated with a length of $7 \mathrm{~m}$ and a diameter of $0.0149 \mathrm{~m}$.

In Figure 8 the results of a simulation with the overall model using the thermodynamic equilibrium model for the post critical heat flux heat transfer are shown. The

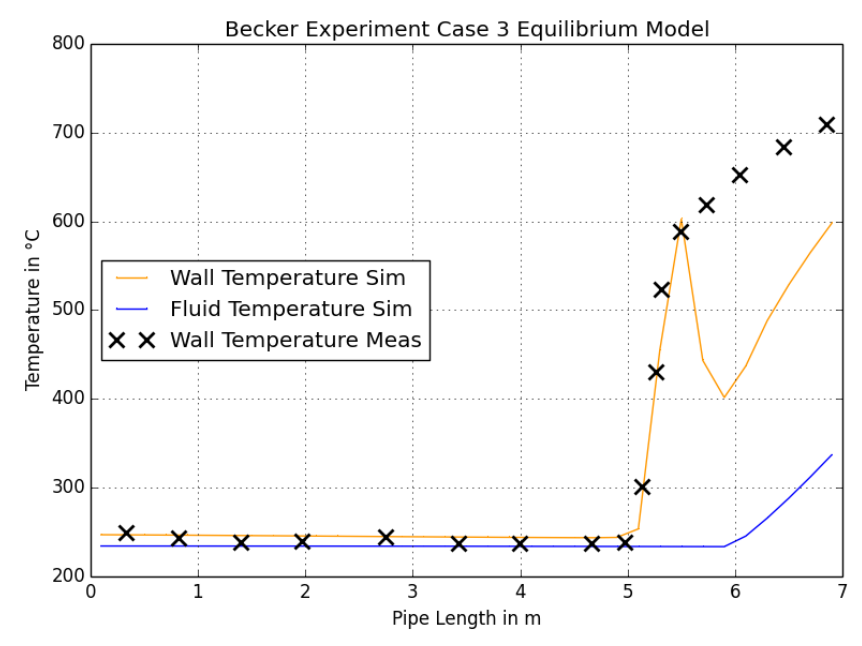

Figure 8. Becker Experiment Case 3 Equilibrium Model

wall temperatures before the heat crisis and the location of the heat crisis are predicted correctly. The predicted wall temperatures after the heat crisis until a length of approximately $5.5 \mathrm{~m}$ match also the measurement. Beyond that point the post critical heat flux heat transfer model is not valid, the overall boiling model changes to the onephase heat transfer correlation. This change leads to an underestimation of the wall temperature, as the gas phase is superheated in the post critical heat flux area. This underestimation has two main reasons. On the one hand the definite temperature is underestimated as the temperature difference for calculation of the heat flux is formed with the equilibrium temperature. On the other hand, the heat transfer coefficient is calculated with fluid properties at the equilibrium temperature, the fluid properties at the superheated gas temperature should be used instead.

In Figure 9 the test case is simulated with the overall model using the thermodynamic non-equilibrium model. The effect of the superheating of the wall near gas phase is included in the non-equilibrium model. Thus, it matches the measurement much better than the simple equilibrium model. At a length of $6 \mathrm{~m}$ the model keeps the wall temperatures constant. This assumption fits also better to the measurement than the use of the one phase heat transfer coefficient as it is done by the simple thermodynamic equilibrium model.

The thermodynamic non-equilibrium model is able to predict the wall temperatures more accurate than the simple equilibrium model. Especially, the transition to the purely convective one phase heat transfer regime fits much better to the experiment data. However, this advantage is dearly bought by a loss of numerical stability and efficiency. This is due to the introduction of a fictive gas 


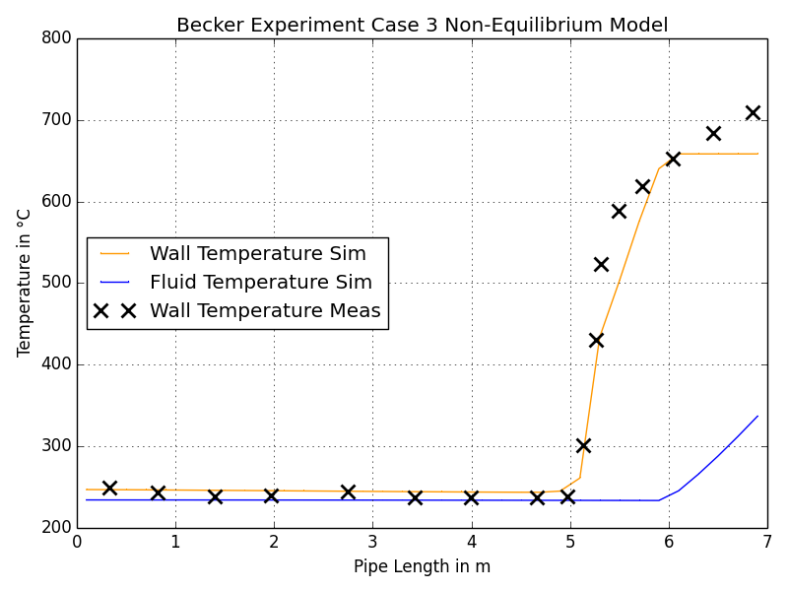

Figure 9. Becker Experiment Case 3 Non-Equilibrium Model

temperature and the indirect manipulation of the wall temperatures. These means are introduced to overcome the drawback of an equilibrium temperature for gas and liquid phase which is used for calculation of the heat balance in the three equation pipe model. For a more accurate modelling of the effects in the post critical heat flux regime a separate energy balancing of the liquid and the gas phase is necessary. This would require a thermo-hydraulic framework which includes volume models for separate balancing of the phases, the so called five or six conservation equation models, see (Hänninen and Ylijoki, 1992).

\section{Summary}

In this paper an implementation of an extended heat transfer model for two phase flow in pipes is presented.

The various correlations of the different boiling regimes are implemented in separate subfunctions. The functional based approach of the FluidDissipation (XRG Simulation) is used. It enables users to create models which are tailored to their problem.

An exemplary application within the thermo-hydraulic framework of the ClaRa library is given. This model is used to verify the implementation. A comparison with a widely used saturated boiling model is conducted. The predicted heat transfer coefficients of the two models differ by magnitudes. These results show the importance of complex heat transfer models in system simulation. These models are relevant in applications, in which crucial variables depend strongly on the heat transfer coefficient, e.g. a safety analysis concerning the pipe wall temperatures.

For validation of the extended heat transfer experiment data from the literature are used. The simulations match the measurement data well. Especially, the position of the critical boiling state is predicted correctly. For more accurate results concerning the post critical heat flux regime a separate balancing of the phases is necessary, which is a feature of the six conservation equations models.

\section{References}

H. Abel-Larsen, A. Olsen, J. Miettinen, T. Siikonen, J. Rasmussen, A. Sjoberg, and K. Becker. Heat transfer correlations in nuclear reactor safety calculations. Technical report, Nordic liaison committee for atomic energy, 1985.

H. Auracher, G. Drescher, D. Hein, O. Herbst, A. Katsaounis, V. Kefer, and W. Köhler. VDI Heat Atlas, chapter Hbc - Kritische Siedezustände. 9th edition, 2002.

D. Biberg. An explicit approximation for the wetted angle in two-phase stratified pipe flow. The canadian Jounal of Chemical Engineering, 1999.

N.O.W.W. Brinkmeier. Flexibilisierung von Kraftwerken. $\mathrm{PhD}$ thesis, Technische Universität Braunschweig, 2015.

J. Brunnemann, F. Gottelt, K. Wellner, A. Renz, A. Thüring, V. Roeder, C. Hasenbein, C. Schulze, G. Schmitz, and J. Eiden. Status of ClaRaCCS: Modelling and Simulation of CoalFired Power Plants with $\mathrm{CO}_{2}$ Capture. Proceedings of the 9th International Modelica Conference, Munich, Germany, pages $609-618,2012$.

J. C. Chen. Correlation for boiling heat transfer to saturated fluids in convective flow. Industrial and Engineering Chemistry Process Design and Development, 5:322-329, 1966.

E.N. Ganic and W.M. Rohsenow. Dispersed flow heat transfer. International Journal of Heat and Mass Transfer, 20:855866, 1977.

D.C. Groeneveld. Post-dryout heat transfer at reactor working conditions. In Proceedings of the National Topical Meeting on Water Reactor Safety. Atomic Energy of Canada Limited, 1973.

D.C. Groeneveld. The 2006 CHF look-up table. Nuclear Engineering and Design, 237:1909-1922, 2007.

K.E. Gungor and R.H.S Winterton. Simplified general correlation for saturated flow boiling and comparisons of correlations with data. Chemical Engineering Research and Design, 1987.

M. Hänninen and J. Ylijoki. The one-dimensional seperate twophase flow model of apros. Technical report, Technical Research Centre of Finland, 1992.

E. Hahne K. Spindler, N. Shen. Vergleich von Korrelationen zum Wärmeübergang beim unterkühlten Sieden. Wärme- und Stoffübertragung, 1990.

A. Katsaounis. VDI Heat Atlas, chapter Hbd -Wärmeübergang nach der Siedekrise. 9th edition, 2002.

Ohno H. Katto Y. An improved version of the generalized correlation of critical heat flux for the forced convective boiling in uniformly heated vertical tubes. Int. Journal for Heat Mass Transfer, 27, 1984.

W. Köhler. Einfluß des Benetzungszustandes der Heizfläche auf Wärmeübergang und Druckverlust in einem Verdampferrohr. $\mathrm{PhD}$ thesis, 1983. 
M.S. Owen, editor. ASHRAE Handbook - Fundamentals, chapter 5 - Two Phase Flow. ASHRAE, 2005.

J.J. Schröder. VDI Heat Atlas, chapter Hba - Strömungssieden unterkühlter Flüssigkeiten. 9th edition, 2002.

M. M. Shah. Chart Correlation for Saturated Boiling Heat Transfer: Equations and Further Study. ASHRAE Transaction1982, $88,1982$.

D. Steiner. VDI Heat Atlas, chapter Hbb - Strömungssieden gesättigter Flüssigkeiten. 9th edition, 2002.

D. Steiner and J. Taborek. Flow Boiling Heat Transfer in Vertical Tubes Correlated by an Asymptotic Model. Heat Transfer Engineering, 13(2):43-69, 1992.

The ClaRa development team. ClaRa - Simulation of ClausiusRankine cycles. URL www. claralib.com. fetched Dec, 15 th 2016.

J. R. Thome, editor. Engineering Data Book III, chapter 10 Boiling Heat Transfer inside Plain Tubes. Wolverine Tube Inc., 2006a.

J. R. Thome, editor. Engineering Data Book III, chapter 18 Post Dry-Out Heat Transfer. Wolverine Tube Inc., 2006b.

T. Vahlenkamp and S. Wischhusen. FluidDissipation for Applications - A Library for Modelling of Heat Transfer and Pressure Loss in Energy Systems. In Proceedings 7th Modelica Conference, Como, Italy, September 2009.

K.E. Gungor R.H.S Winterton. A general correlation for flow boiling in tubes and annuli. Int. J. Heat Mass Transfer, 29(3): 351-358, 1986.

XRG Simulation. URL http://www.xrg-simulation. de/de/produkte/xrg-library/

xrg-fluiddissipation-library. fetched Dec., 15th 2016. 